\title{
Optimisation of Frame-Shaped Fluxgate Sensor Core made of Amorphous Alloy Using Generalized Magnetostatic Method of Moments
}

\author{
R. SZEWCZYK ${ }^{a, *}$ AND P. FRYDRYCH ${ }^{b}$ \\ ${ }^{a}$ Industrial Research Institute for Automation and Measurements, Al. Jerozolimskie 202, 02-486 Warsaw, Poland \\ ${ }^{b}$ Institute of Metrology and Biomedical Engineering, Warsaw University of Technology, \\ św. A. Boboli 8, 02-525 Warsaw, Poland
}

\begin{abstract}
Fluxgate magnetic field sensors are commonly used in the industry, navigation as well as in non-destructive testing. Moreover, recent development of such sensors is focused on effective application of thin layer magnetic cores, such as made of amorphous alloys. However, effective development of fluxgate sensors require method of modeling its core taking into account demagnetization. The paper presents the results of optimization of the shape of thin-layer core for fluxgate sensor. Due to the fact that possibility of application of finite elements method is limited in the case of thin layer, the method of moments was used. Considering the geometry of the core as well as magnetizing and sensing winding, the optimal proportion of the length/thickness parameter was determined from the point of view of sensor sensitivity. It was proven that value of this optimal proportion is strongly dependent of thickness of the core.
\end{abstract}

DOI: 10.12693/APhysPolA.131.660

PACS/topics: $85.75 . \mathrm{Ss}, 75.50 . \mathrm{Kj}$

\section{Introduction}

Fluxgate sensors are commonly used in industry and scientific research due to their high sensitivity, low noise level as well as the wide range of operating temperature [1]. Recently developed fluxgate sensors often use thin layers of magnetic materials as its inductive core [2]. Especially amorphous magnetic alloys are very attractive materials for single [3] and two axis [4] fluxgate sensors developed as a multi-layer printed boars circuit (PCB).

It should be stressed that for fluxgate sensors, the signal-to-noise ratio is the most important. However, sensor sensitivity is one of the most contributing factor in the signal-to-noise ratio. Moreover, in the case of $\mathrm{PCB}$ sensors, other parameters influencing on the signal-tonoise ratio, such as quality of the edges or properties of the material, are independent of the sensitivity caused by the sensor geometry and can analyzed separately.

In spite of promising applications, the guidelines for the shape of frame core for two axis PCB-based fluxgate sensors were not presented in the literature. The paper is filling this gap presenting the method and the results of optimization process of the frame-shaped core of fluxgate sensor focusing on the sensor sensitivity.

\section{Two axis fluxgate sensors as PBCs}

General view of two-axis fluxgate sensor developed as the PBC [5] is presented in Fig. 1a. Such sensor utilizes the frame-shaped inductive core (presented in Fig. 1b)

\footnotetext{
*corresponding author; e-mail: rszewczyk@onet.pl
}

made as an amorphous alloy layer, with thickness $t$ typically equal to $30 \mu \mathrm{m}$.
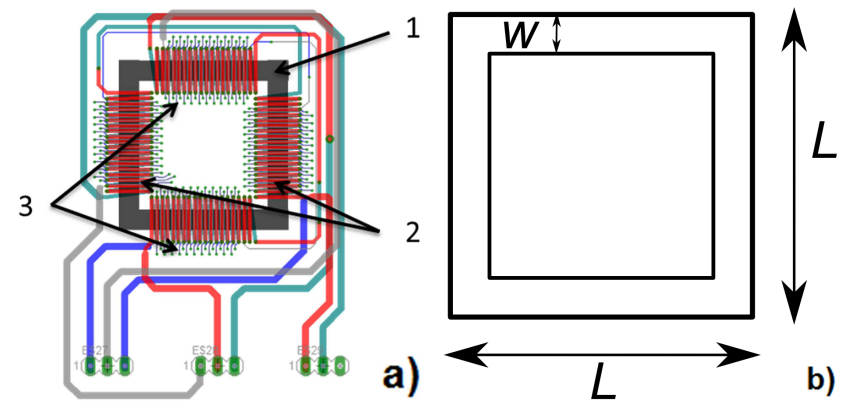

Fig. 1. Two axis PBC fluxgate: (left) general view of the sensor: (1) frame-shaped core, (2) magnetizing winding, (3) sensing winding, (right) frame-shaped core of the sensor made of amorphous alloy thin layer.

Length of the frame-shaped core is described as $L$, whereas its width is marked $w$. On each side of the frameshaped core, both magnetizing and sensing winding is wound. In such a case, the output signal $U(t)$ from the sensor is given by the following equation:

$$
U(t)=n \frac{\mathrm{d} B(t)}{\mathrm{d} t} w t,
$$

where $n$ is the number of turns of sensing winding, and $B$ is the flux density in the core.

\section{Optimization of the shape of the core}

During the magnetization of frame-shaped core of the fluxgate by the external, measured magnetizing field $H_{m}$, achieved value of flux density $B_{m}$ in the core is determined mainly by the demagnetization process than by the relative permeability of the core material. Calculation of 
the distribution of flux density $B_{m}$ in the core is not a trivial task. Due to the problems with application of finite elements method for this purpose (connected with the radical increase of the number of elements in the mesh for the thin layers [6-8]), the method of moments should be applied. Generalization of the method of moments for thin layers is presented elsewhere [9].
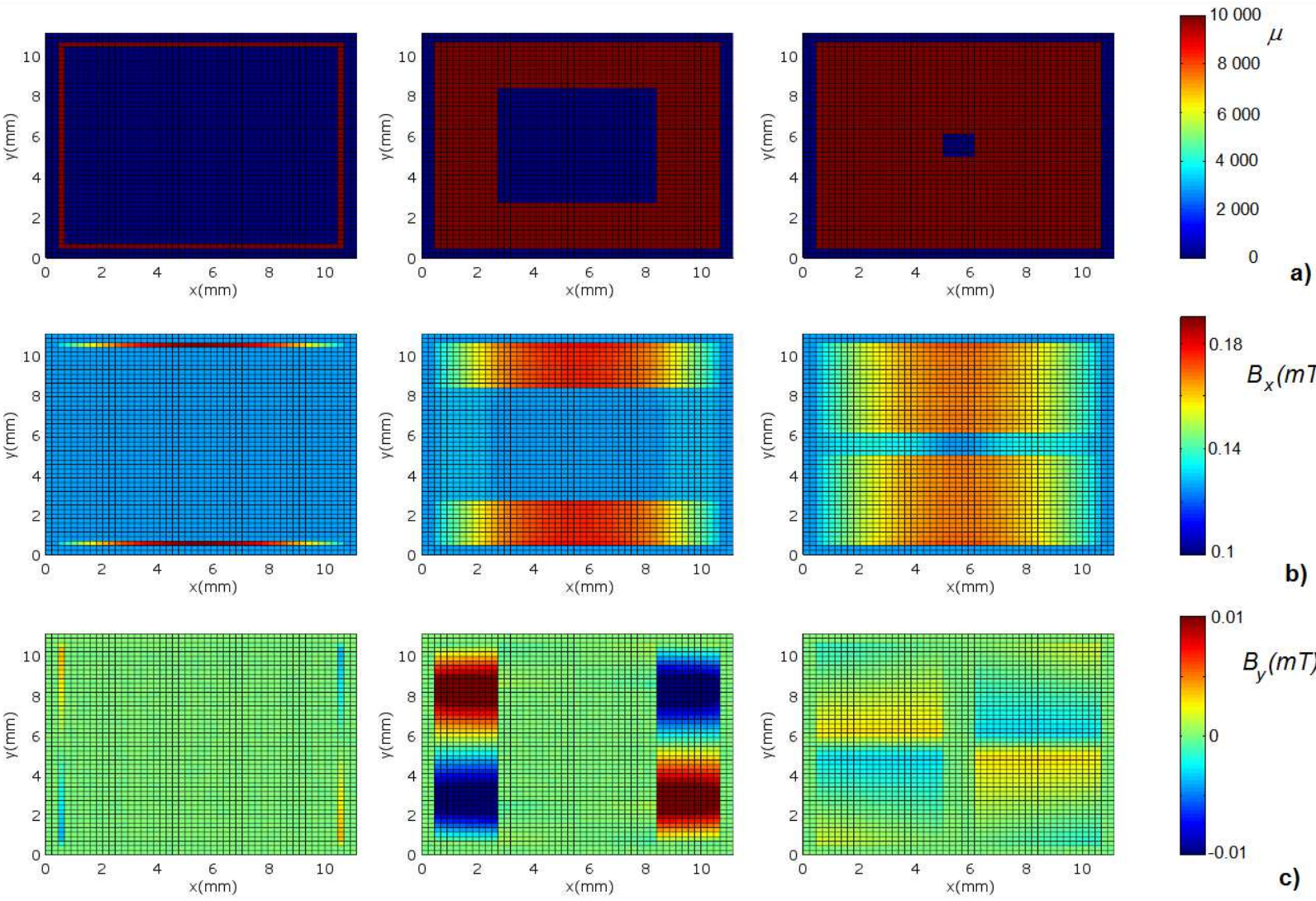

Fig. 2. The results of simulation for frame-shaped core with thickness $t$ equal $30 \mu \mathrm{m}$ and uniform square mesh (for the different values of $2 w / L$ ): (a) distribution of permeability, (b) distribution of the flux density $B$ in the $x$ direction, (c) distribution of the flux density $B$ in the $y$ direction.

Figure 2 presents the results of simulation performed by the method of moments for thin layer, frame-shaped core with length $L$ equal to $10 \mathrm{~mm}$, thickness $30 \mu \mathrm{m}$ and material relative permeability $\mu=10000$. Simulation was carried out for three values of core width $w$ and magnetizing field $H$ equal to $100 \mathrm{~A} / \mathrm{m}$. Stronger demagnetization effect may be observed for higher values of core width $w$. This phenomenon is confirmed by Fig. 3 presenting the distribution of the flux density $B$ in the middle of frame-shaped cores side, during the magnetization by uniform value of external magnetizing field $H$ in $x$ direction. Results are presented for different values of parameter $2 w / L$ describing the core. Moreover, the results of simulation show that for core material relative permeability $\mu$ changing from $10^{3}$ to $10^{5}$, the value of flux density in the core changes less than $0.3 \%$. It clearly shows that for the analysed shapes of the core, flux density $B$ distribution in the core is determined by the demagnetization caused by the shape of the core.

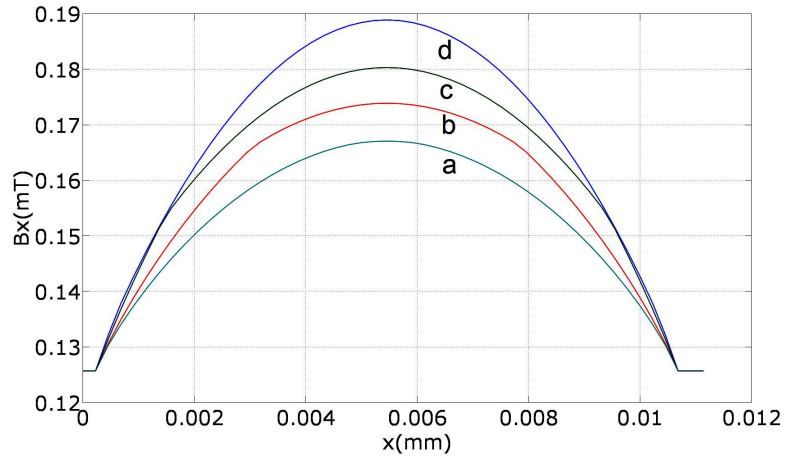

Fig. 3. Flux density $B$ in the middle of frame-shaped cores side, during the magnetization by uniform external magnetizing field $H=100 \mathrm{~A} / \mathrm{m}$ (material permeability $\mu=10000$, thickness $t=30 \mu \mathrm{m}$ ). Simulation done for different values of $2 w / L$ core parameter: (a) 0.05 , (b) 0.23 , (c) 0.55, (d) 0.91 . 
On the base of presented results of simulations, the optimization of $2 w / L$ frame-shaped core parameter may be carried out. For optimization, the following assumptions should be stated:

- thickness of the core is equal to $30 \mu \mathrm{m}$, and its length $L$ is $10 \mathrm{~mm}$. The core is magnetized by the external magnetic field $H=100 \mathrm{~A} / \mathrm{m}$,

- sensing winding is wound at the length $L-2 w$ $1.8 \mathrm{~mm}$ of the central part of the internal side of the frame-shaped core with given turns per millimeter winding density. Value of $2 \times 0.9 \mathrm{~mm}$ margin in the sensing core is caused by the technological requirements,

- output signal is proportional to the average value of flux density $B$ from the external magnetic field $H$ (which is true due to the principles of operation of a fluxgate sensor [10]). As a result, the magnetostatic simulations were carried out.

The results of optimization are presented in Fig. 4. It can be seen that value of output signal $k$ (given in arbitrary units) has maximum at $2 w / L$ equal to 0.375 . Moreover, it tends to zero for very narrow core $(2 w / L \rightarrow$ 0 ) and when there is no place for the sensing winding $(2 w / L>0.6$, the inside hole is too narrow for the sensing winding).

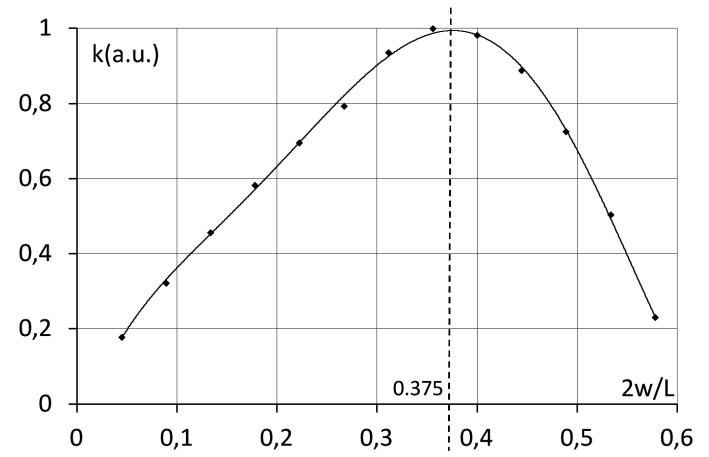

Fig. 4. The results of optimization of the $2 w / L$ parameter of the frame-shaped core. Maximal output signal $k$ is reached for $2 w / L$ equal to 0.375 .

Presented optimization was carried out for the given value of frame-shaped core thickness $t$ equal to $3 \mu \mathrm{m}$. This value of thickness is typical for frame-shaped cores produced from the rapidly quenched amorphous alloys during the photolithography process. However, presented method may be used also for other core thickness, especially for ultrathin layers produced for microsensors. However, in such a case optimal value of $2 w / L$ may be different due to the different demagnetization of the core.

\section{Conclusions}

Optimization of the thin layer cores of fluxgate sensors requires magnetostatic simulations of flux density $B$ distribution in the core. However, due to the fact that finite elements method is not suitable for such simulation, the application of previously presented, generalization of the method of moments for thin layers was used.

In the case of frame-shaped fluxgate core made of amorphous alloy with length $L$ equal to $10 \mathrm{~mm}$ and thickness $30 \mu \mathrm{m}$, the highest sensor sensitivity is reached for $2 w / L$ equal to 0.375 . Value of optimal $2 w / L$ does not depend of relative permeability of the core, when it vary from $10^{3}$ to $10^{5}$. However, other values of core thickness, optimization process should be carried out again. Repeating of optimization calculations is necessary due to the different value of demagnetization of the core and variations in the distribution of flux density $B$.

\section{References}

[1] P. Ripka, Sens. Actuat. A Phys. 33, 129 (1992).

[2] P. Ripka, Sens. Actuat. A Phys. 37-38, 417 (1993).

[3] J. Kubik, L. Pavel, P. Ripka, Sens. Actuat. A Phys. 130-131, 184 (2006).

[4] P. Kejı̌k, L. Chiesi, B. Janossy, R.S. Popovic, Sens. Actuat. A Phys. 81, 180 (2000).

[5] P. Frydrych, R. Szewczyk, J. Salach, K. Trzcinka, IEEE Trans. Magn. 48, 1485 (2012).

[6] Y. Vuillermet, M. Audoin, R. Cuchet, Sens. Actuat. A Phys. 158, 212 (2010).

[7] O. Chadebec, J.-L. Coulomb, J.-P. Bongiraud, G. Cauffet, P. Le Thiec, IEEE Trans. Magn. 38, 1005 (2002).

[8] R. Szewczyk, in: Proc. APCOM 2016 Conf., Štrbské Pleso, (Slovak Republic), 2016, Eds.: J. Vajda, I. Jamnický, p. 178.

[9] R. Szewczyk, Acta Phys. Pol. A 131, 845 (2017).

[10] F. Primdahl, IEEE Trans. Magn. 6, 376 (1970). 